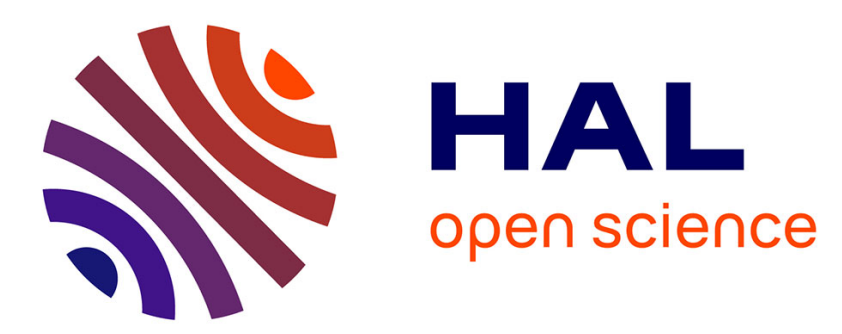

\title{
Anterior cingulate activity during routine and non-routine sequential behaviors in macaques.
}

Emmanuel Procyk, Yuji L. Tanaka, Jean-Paul Joseph

\section{To cite this version:}

Emmanuel Procyk, Yuji L. Tanaka, Jean-Paul Joseph. Anterior cingulate activity during routine and non-routine sequential behaviors in macaques.. Nature Neuroscience, 2000, 3 (5), pp.502-8. $10.1038 / 74880$. hal-00131425

\section{HAL Id: hal-00131425 \\ https://hal.science/hal-00131425}

Submitted on 16 Feb 2007

HAL is a multi-disciplinary open access archive for the deposit and dissemination of scientific research documents, whether they are published or not. The documents may come from teaching and research institutions in France or abroad, or from public or private research centers.
L'archive ouverte pluridisciplinaire HAL, est destinée au dépôt et à la diffusion de documents scientifiques de niveau recherche, publiés ou non, émanant des établissements d'enseignement et de recherche français ou étrangers, des laboratoires publics ou privés. 


\title{
Anterior cingulate activity during routine and non-routine sequential behaviors in macaques
}

\author{
E. Procyk ${ }_{1,2}$, Y. L. Tanaka ${ }_{3}$ and J. P. Joseph 1 \\ 1 INSERM, Unité 94: Espace et Action, 16 av Lépine, Case 13, 69676 Bron, France \\ 2 current address: Yale University School of Medicine, Section of Neurobiology, 333 Cedar Street, SHM B413, New Haven, Connecticut \\ 06510, USA \\ з Department of Physiology, Nihon University School of Medicine, 30-1, Oyaguchi-Kamicho, Itabashi-Ku, Tokyo 173, Japan
}

Anterior cingulate cortex is important in monitoring action for new challenges. We recorded neuron activity in the anterior cingulate sulcus of macaques while they performed a sequential problem-solving task. By trial and error, animals determined the correct sequence for touching three fixed spatial targets. After the sequence was repeated three times, we then changed the correct solution order, requiring a new search. Irrespective of component movements or their kinematics, task-related neurons encoded the serial order of the sequence. Neurons activated with sequence components $(68 \%)$ differed in activity between search and repetition. Search-related activity occurred when behavioral flexibility was required and ended as soon as the animal accumulated enough information to infer the solution, but had not yet tested it. Repetition-related activity occurred in a regime of memory-based motor performance in which attention to action is less necessary.

Several theories suggest that non-routine behaviors (learning, problem solving) involve different brain regions than do routine behaviors (automatic responses in welllearned situations)1,2. Functional imaging studies suggest that one of the structures involved in non-routine behaviors is the anterior cingulate cortex (ACC). For example, a rostral cingulate zone is selectively activated during sequence learning and conditional association learning ${ }_{3,4}$. Activation of the rostral ACC is also consistently observed when humans are free to select their own responses. This suggests a role for ACC in attention to action and in the selection of appropriate responses to new situations3-6. The rostral ACC is also thought to be specifically involved in monitoring the competition between different response tendencies in situations where errors are likely to occur7. The rostral ACC in humans is thought to correspond to the anterior portion of the cingulate sulcus in nonhuman primates8- 10. To date, however, there are few neurophysiological studies of this area in monkeys, although single-neuron recordings in the rostral part of the cingulate sulcus of monkeys show activity specific to motor set for self-paced movements and to voluntary movement selection based on reward 11,12 .

Here we recorded the activities of single neurons in the dorsal bank of the anterior portion of cingulate sulcus (termed ACd) as rhesus monkeys performed a sequential problem-solving task. The task required the monkeys to discover by trial and error a particular sequence for touching three targets. Having discovered the correct sequence, they had to repeat it three times. The monkeys received feedback on each touch, which allowed them to infer the correct response and then repeat it; we were therefore able to monitor activity in the ACd as the monkeys' behavior evolved from trial-and-error exploration to routine execution of the correct solution. We report that $A C d$ neuron activities had two important properties. First, they encoded the serial order of movements in sequences with little regard to component movements or to their kinematics, and second, activities differed in trial-and-error exploration and routine performance.

\section{RESULTS}

Behavioral task

We obtained data from two monkeys. A trial began with the simultaneous onset of three targets on a touch screen in front of the monkey; the locations of these targets (which we designate upper, left, right) remained fixed throughout the experiment (Fig. 1). The animal's task was to select each target in turn, in the correct sequence. There were six possible sequences; the experimenter randomly selected the correct solution, and the animal had to discover it by trial and error. For each target selected, the monkey was required to fixate it with an eye movement and then touch it with the hand. If the first choice was correct, the animal proceeded to the next; if this was also correct, it proceeded to the third (which, in principle, could then be inferred by elimination).Correct choices were confirmed with a brief sound cue. If the animal made an incorrect choice, the targets disappeared from the screen, the trial was aborted, and the animal had to begin a new trial from the first touch. (The correct solution remained unchanged.) After the first correct trial, the monkey was rewarded with a drop of juice. It was then required to repeat the correct sequence three more times and was rewarded each time. After the last 


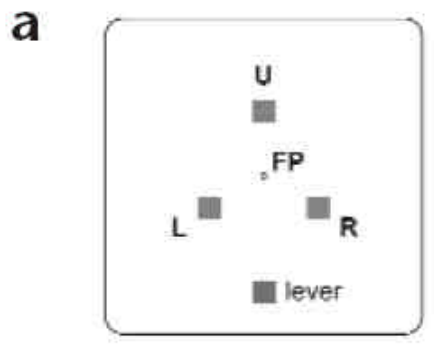

b

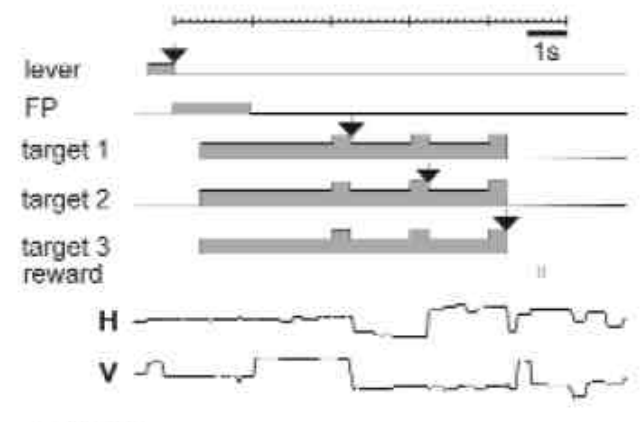

: touch

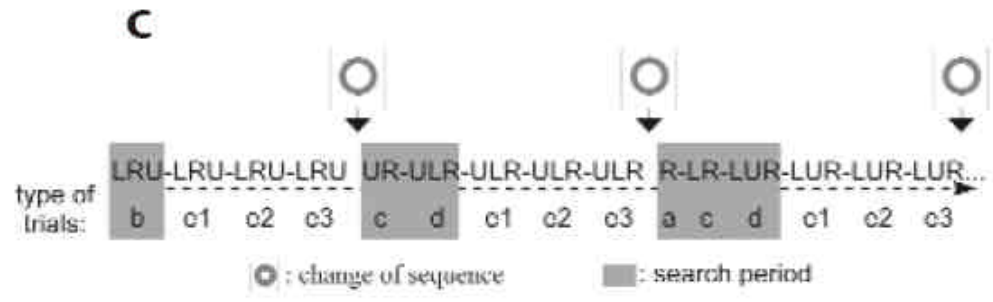

d

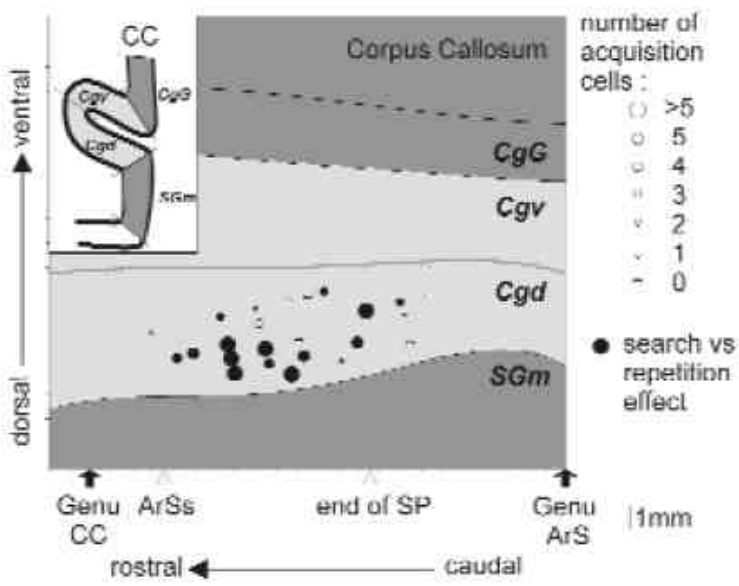

e

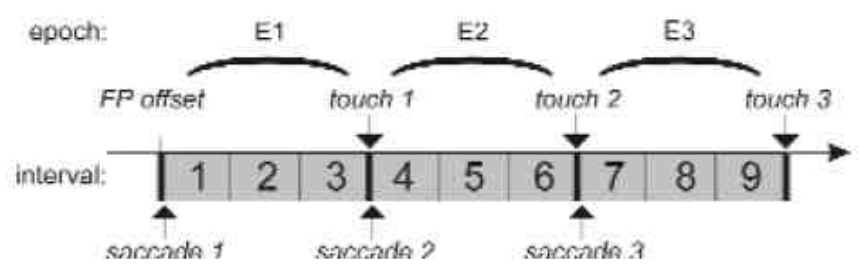

Fig. 1. Display, trial events, trial structure, anatomical location of task-related neurons in the problem-solving task and taskrelated intervals. (a) Location of stimuli on the display monitor. The animal worked with three targets, upper (U), left (L) and right (R). A 4 ' $4 \mathrm{~mm}$ central white square served as fixation point (FP). A $2 \mathrm{~cm}$ ' $2 \mathrm{~cm}$ red square located $10 \mathrm{~cm}$ below the horizontal center served as the starting lever. (b) Schema of trial events. The animal was trained to search for the correct order of the three targets. Each correct touch (black arrow) was signaled by a short tone delivered from a buzzer located above the screen. The monkey was rewarded only after three correct touches. In case of an incorrect touch, a break of fixation or an early touch, all targets were extinguished, and the trial was aborted. Gray area, time of illumination of the lever, the FP and the three targets. Higher amplitude in the gray area: full illumination (GO signal). Representative eye movements for a sequence U-L-R are shown $(\mathrm{H}$, horizontal eye position; $\mathrm{V}$, vertical eye position). (c) Example of a chronological list of trials performed during search and repetition of three successive sequences ( $L-R-U, U-L-R$ and $L-U-R)$. Below are indicated the types of trials executed by the animal (see Methods); trials of search periods are represented on a gray background. The signal indicating a change of sequence is schematized by a circle in a square. (d) Two-dimensional reconstruction of the medial cortex of one monkey. Recording sites in the cingulate sulcus are represented by symbols. Black circles show sites at which cells with the 'search versus repetition effect' were recorded. CC, corpus callosum; ArSs, rostral extent of the superior branch of the arcuate sulcus; ArS, arcuate sulcus; end of SP, caudal extent of sulcus principalis; SGm, medial superior gyrus; Cgd and Cgv, dorsal and ventral banks of cingulate sulcus; CgG, cingulate gyrus. (e) Task-related intervals (1-9; see Methods) of a trial.

correct trial, a combined visual and auditory cue indicated to the monkey that a new sequence had been selected. The period up to and including the first correct performance of the sequence is referred to as the 'search period'. The subsequent period during which the correct sequence was repeated three times was the 'repetition period'. The animals were already familiar with the requirements of the task and with the six possible solutions before the experiment began. Thus, our experiment was not concerned with general learning processes; rather, our aim was to examine the rapid adaptation of responses under the guidance of error feedback. The monkeys could solve up to 60 sequences during a single recording session. Behavioral data obtained during the recording sessions indicate that they conducted a methodical search for the correct solution and rarely repeated incorrect touches. For example, if the first target was correct and the second was 


\begin{tabular}{|c|c|c|c|c|c|}
\hline & $\begin{array}{l}\text { Post-response } \\
\text { Intervals I, 4, } 7\end{array}$ & $\begin{array}{c}\text { Delay } \\
\text { Intervals 2, 5, } 8\end{array}$ & $\begin{array}{c}\text { Pre-response } \\
\text { Intervals 3, 6, } 9\end{array}$ & Total & \\
\hline Order & $86(69 \%)$ & $84(67 \%)$ & $97(78 \%)$ & \multirow[t]{2}{*}{$267(71 \%)$} & \multirow{4}{*}{$\begin{array}{c}\chi^{2} \\
p<0.0001\end{array}$} \\
\hline & & NS at $p<0.05, \chi^{2}$ & & & \\
\hline Origin & $33(27 \%)$ & $35(28 \%)$ & $27(22 \%)$ & \multirow[t]{2}{*}{$95(26 \%)$} & \\
\hline & & NS at $p<0.05, \chi^{2}$ & & & \\
\hline End point & $28(22 \%)$ & $26(2 \mid \%)$ & $25(20 \%)$ & 79 (21\%) & \\
\hline
\end{tabular}

Post and Pre indicate post- and pre-response intervals (Fig. I). For each type of interval, the number and proportions of cells are indicated (total number of cells, 125). Total numbers of cases with significant effects and the corresponding proportion (in parentheses) of cells are given in the right column (Total).

incorrect, the monkey typically returned in the next trial to the correct first target and changed the second target 13 . An ideal subject would require on average 2 trials to reach the correct solution; monkey 1 found the solution after an average of 2.15 trials (mean of 25 recording sessions) and monkey 2 after 2.86 trials (10 sessions). Overall, $95 \%$ of the searches were optimal. Although we were interested in the underlying cognitive processes rather than in the monkey's movements per se, it was important to know whether any of the observed changes in neuronal activity could be attributed to small changes in movement parameters. We therefore measured reaction times (RT; the time to initiate a hand movement), movement times (the time taken to complete the movement by touching the screen) and timing of saccades that were typically made toward the next target. In monkey 1 , reaction times were consistently shorter during repetition than during the search period. Both RTs and movement times (MTs) were shorter for the second and third movements than for the first; this was true for both the search and the repetition periods. In monkey 2, MTs were consistently shorter during repetition than during search. For both periods, RTs and MTs were shorter for the third movement than for the first and second movements. We did not obtain eye movement data for monkey 2, but, during each interval of free oculomotor activity (see methods), monkey 1 made on average 1.30 orientating saccades in the search periods and 1.18 during repetition periods. The relative timing of target touches and onsets of saccades to the next target also differed between search and repetition periods. During search, the saccade to the next target was almost always made after the target touch (Fig. 2). During repetition, the saccade to the next target often preceded the target touch. There were no differences in eye stability - measured by the number of breaks of fixationbetween the search and repetition periods.

We recorded neural activity from the anterior part of the dorsal bank of the cingulate sulcus. The recording sites were located at rostrocaudal levels anterior to the genu of the arcuate sulcus and posterior to the anterior extent of the superior branch of the arcuate sulcus (Fig. 1d). This dorsal part of the anterior cingulate sulcus is adjacent to the pre-supplementary motor area (pre- SMA) and is considered to be a subfield of area $24 \mathrm{c}$ (refs. 14-16). We recorded from 191 task-related neurons. Among these, 65 neurons $(34 \%)$ demonstrated activity changes related only to the onset of targets, rewards or error cues. Because we were primarily concerned with motorrelated activity, these neurons are not considered further here. Activity of the remaining 125 neurons $(66 \%$; 109 in monkey 1 on both sides and 16 in monkey 2 , on one side only) was related to the monkey's behavior, changing activity during the period when the monkey was making eye or hand movements toward the targets. Our study focused on these neurons.

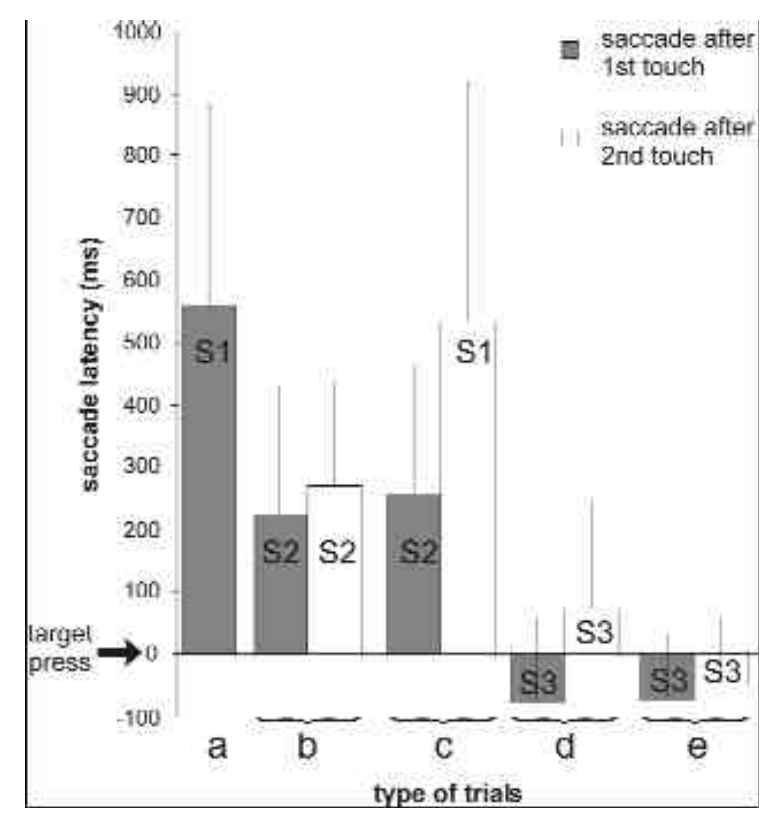

Fig. 2. Saccadic RTs after the first and second target touches in different types of trials (one monkey). S1 and S2, saccades after incorrect and correct touches during search; S3, saccades during repetition. Mann- Whitney U-test, S1 versus $\mathrm{S} 2, p<10-7$; S2 versus S3, $p<10-7$.

\section{Encoding of serial order}

Each trial began with the appearance of a fixation point (FP), whose disappearance after two seconds was the cue for the monkey to begin the task. During the subsequent period, which we refer to as 'target acquisition', the monkey was required to make an eye movement to the target and fixate it until the monkey received a GO signal (a transient increase in brightness of the entire target array) instructing it to touch the target. If the choice was correct, an auditory cue instructed the monkey to proceed immediately to the next eye movement. For the purpose of analyzing the data, we divided the target acquisition period into three epochs (E1, offset of FP to first target touch; E2, first touch to second touch; E3, second touch to third touch). Each of these epochs was further divided into three task-related intervals of approximately equal 


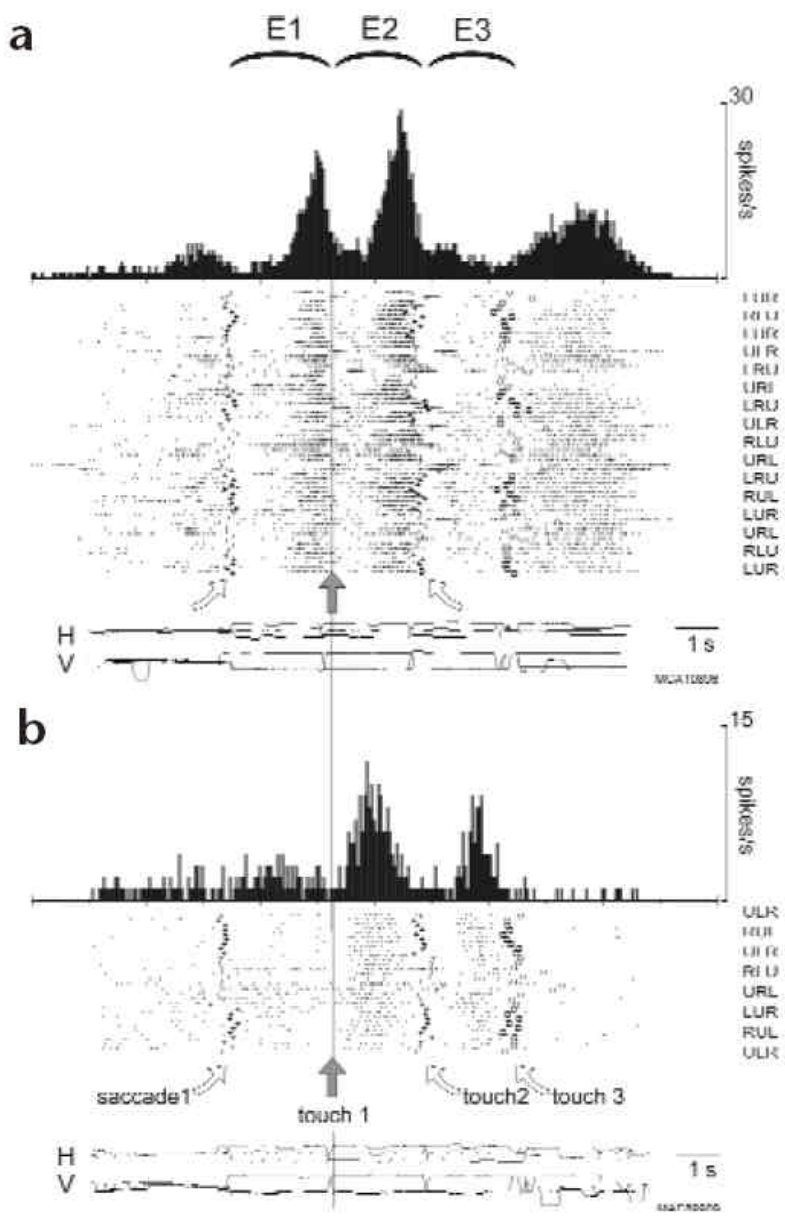

duration, for a total of nine intervals in each completed trial (Fig. 1e). These were 'post-acquisition' (the $600 \mathrm{~ms}$ following FP offset or target touch; intervals 1, 4, 7), then 'delay' (from $t=600 \mathrm{~ms}$ until the GO signal, a variable interval of between 850 and $950 \mathrm{~ms}$; intervals 2, 5, 8) and lastly 'pre-acquisition' (from the GO signal until target touch, typically $750 \mathrm{~ms}$; intervals $3,6,9$ ). For each type of interval, we analyzed differences in neuronal activity for correlations with spatial variables (the locations of the targets corresponding to the start and end points of the movements) and serial order. The strongest correlation was with serial order (Table 1). In 37 neurons (30\%), it was the only determinant of activity changes in at least one type of interval (pre- acquisition, delay or post-acquisition). In 80 neurons (64\%), activity changes were correlated with both serial order and spatial variables. We detected neither spatial nor order effects in 8 neurons (6\%). We also examined the influence of saccade parameters (direction, number and latency of saccades) in 55 cells, $85 \%$ of which $(n=46)$ showed a serial-order effect (with RTs and MTs as covariates). When saccade parameters were included, the serial- order effect was still observed in $75 \%$ of cells $(n=$ 41). This result indicates that, for most cells, saccade parameters had only a minor influence on the serial-order effect. Two examples are illustrated; one cell was active mainly in the pre-acquisition intervals preceding the first and second touches, regardless of hand and target positions (Fig. 3a). The other cell (Fig. 3b) was active mainly during the delay intervals preceding the second and (to a lesser extent) third touches.

Fig. 3. Serial-order effects: two examples. Neuronal activities are displayed only for correct trials corresponding to the sequences listed to the right of rasters (four trials per sequence). Each raster line, where a tick represents the occurrence of a single spike, displays cell activity recorded during one trial. Trials are displayed in chronological order (from top to bottom) and correspond to groups of successive correct trials. The third touch is indicated in each trial by an empty square. Rasters and histograms are aligned on the first touch. Time scales and activity scales are indicated on the right. (a) Activation is primarily observed in the first and second 'pre-acquisition' intervals, not in the third (order effect, $F_{2,187}=104.19, p<10-7$; order effect with saccade parameters as covariates $F_{2,184}=100.96, p<10-7$; spatial effect (origin), $F_{2,123}=0.32, p<0.72$; spatial effect (endpoint), $F_{2,187}=2.53, p<0.09$ ). (b) Activation was observed primarily during the second 'delay' interval (order effect, $F 2,78=26, p<10-7$; with saccade parameters as covariates, $F_{2,78}=19.35, p<10-7$; spatial effect (origin), $F_{2,51}$ $=2.78, p<0.47$; spatial effect (endpoint), $F_{2,78}=3, p<$ $0.055)$.

\section{Search- and repetition-related activity}

Interval-specific activity was also tested for correlations with search versus repetition-that is, we compared equivalent intervals for trials in the search or repetition periods. We examined 1125 intervals in 125 cells ( 9 ' 125$)$; of these, 226 intervals in $87(68 \%)$ cells were differentially active in the two periods. For the majority of these neurons (68 of 87 , or $78 \%$ ), the effect of period was in the same direction for each interval. For instance, if the firing rate during interval 1 was greater for search trials than for repetition trials, the firing rate during interval 2 was also greater for search trials. These neurons could thus be classified as 'search-preferring' (40 cells) or 'repetition preferring' (28 cells) without regard to their possible preferences for particular intervals (Figs. 4 and 5). We also examined the effect of saccade parameters (direction, number, latency) on 55 cells, of which $89 \%$ ( $n=49$ ) showed period-related activity. When saccade parameters were also included as covariates, the period effect was still observed in $78 \%$ of cells $(n=43)$ and disappeared in only 6 cells. We conclude that the period effect, like the serialorder effect described above, is largely independent of any variation in saccade parameters.

\section{Transition from search to repetition}

To analyze the transition between search and repetition, different types of trials were considered: 'a' refers to trials in which the first touch was incorrect and which, therefore, were aborted; 'b' refers to trials that were successful by chance; 'c' refers to trials in which the second touch was incorrect, which were also aborted; ' $d$ ' refers to the correct trials that immediately followed the ' $c$ ' trials, and 'e' refers to the 
a Successive trials

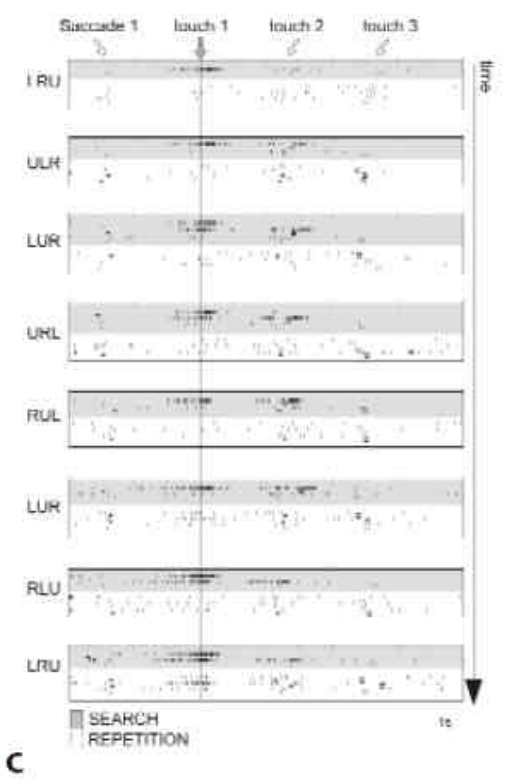

C
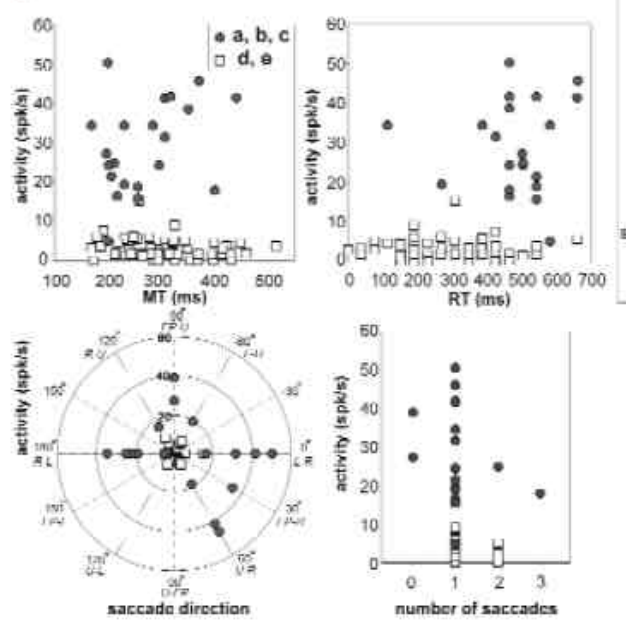

b Type of trials (a,b,c,d, and e)
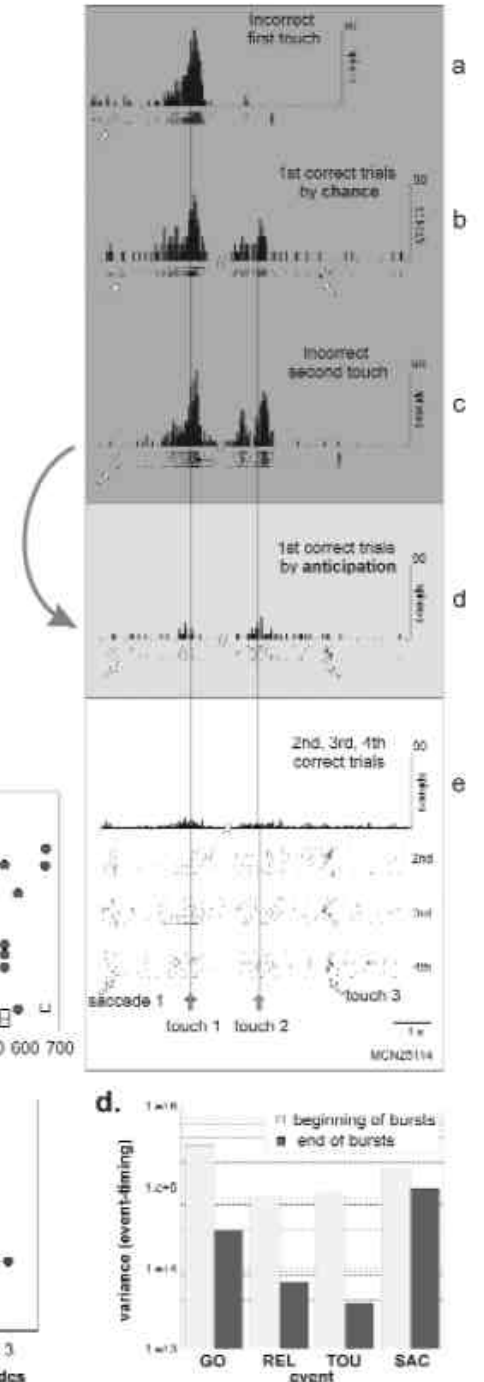

Fig. 4. Search versus repetition differences. Activity of a 'search-preferring' cell. In (a) the neuronal activity in successive trials for each sequence indicated on the left is shown during the search period up to the first correct trial (in gray) and during the three subsequent repetitions of the sequence (in white). Rasters are aligned on the first touch. In (b), the activity of

the same cell is sorted according to the type of trials (see Fig. 1c). The discharge pattern varied from the search to the repetition period. ANCOVA (RT, MT, DEG, NB and SLAT as covariates; interval 4, $F_{1,36}$ $=69.59, p<10-5$; interval $7, F_{1,30}=51.09$,

$p<10-5)$. Note that the cell activity during the ' $d$ ' and 'e' trials is statistically the same ('d' versus 'e' trials; interval $4, F_{1,23}=0.31, p$ $<0.58$; interval $7, F_{1,22}=3.20, p<0.09$ ). In (c), the neural activity of intervals 4 and 7 in ' $a, b, c$ ' and ' $d$, e' trials is plotted against the corresponding RTs, MTs, saccade directions (polar plot) and numbers of saccades. (Number of saccades equal to zero corresponds to incorrect trials at the end of which no saccade was made until the end of spike acquisition.) No significant correlation was found between activity and RT, MT or number of saccades (Spearman test). In (d), variances of neuronal modulation times (bars) during the search period relative to the times of different events (GO, REL, TOU and SAC). The ends of bursts are significantly better linked to the times of target touches (TOU versus GO, $p<10-4$, F-test; TOU versus REL, NS; TOU versus SAC, $p<10$ 4).

Relationship of activity to sensory and motor events

Timing of neuronal discharges was computed for 40 cells for which the onset and offset of neuronal responses were easily identified 27 . In 7 cells

correct trials that corresponded to repetitions (Fig. 1c). Theoretically, the correct solution could always be anticipated after a correct or incorrect second touch and before the first reward. If the second touch was correct by chance ('b' trials), then the third target was obvious. If the second touch was incorrect (' $c$ ' trials), then the solution could be found in the next trial by keeping the first target and changing the second ('d' trials). Importantly, neurons that showed a preference for search or repetition periods always showed transitions in their behavior after the second touch-in other words, after the monkey had obtained enough information that the whole sequence could be anticipated. In the 'b' trials, the activity after the touch becomes transitional or analogous to that observed after the second touch during the 'e' trials (Fig. 4 and 5). In the ' $d$ ' trials, the activity becomes similar to that observed during the ' $e$ ' trials (Figs. 4, 5 and 6). Knowledge of the first target alone was not sufficient to produce a search/repetition transition in the response to this target.
$(17.5 \%)$, the $F$-test indicated that times of neuronal modulation were more related to GO signals, lever and target release, screen touch and/or sensory feedback associated with the touch than to the saccades (Fig. 4d). In the remaining cells, all events were equivalent.

\section{DISCUSSION}

\section{Serial-order effects}

Our data show that the ordinal position (first, second or third) of a target acquisition or of a target fixation affected the activity of the task-related neurons. This effect was independent of orderdependent differences in the manner of performance of arm movements or saccades. Previous studies show that the serial order of sensory stimuli may be encoded in the primary motor cortex, the superior arcuate area and the caudate nucleus17-19. Sequence-related activity can reflect the relational order of specific ocular fixations 
a

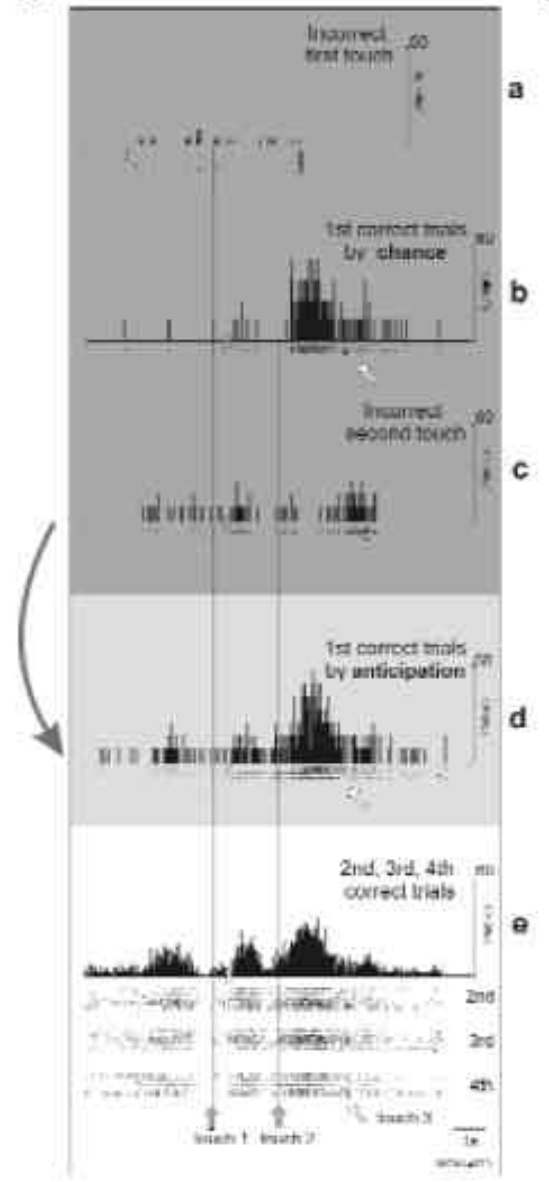

b
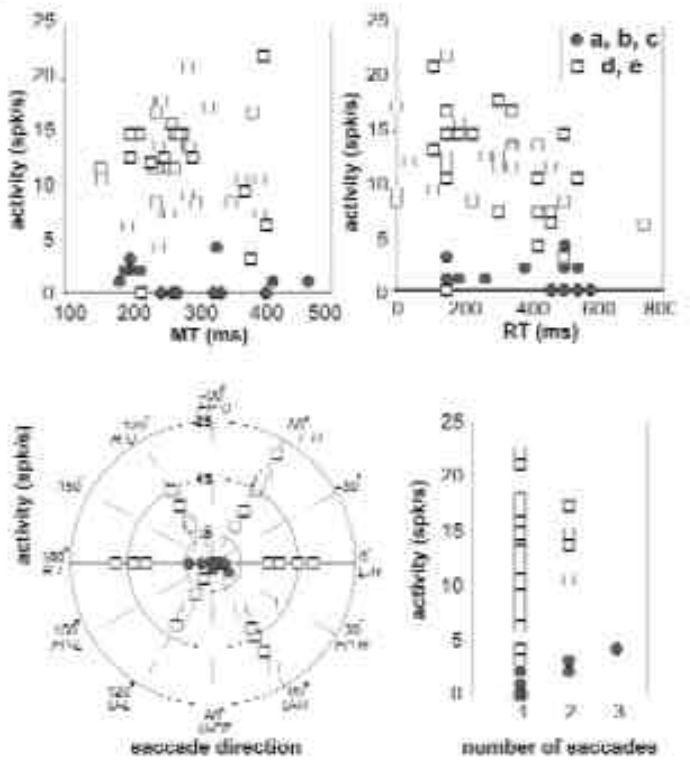

c.

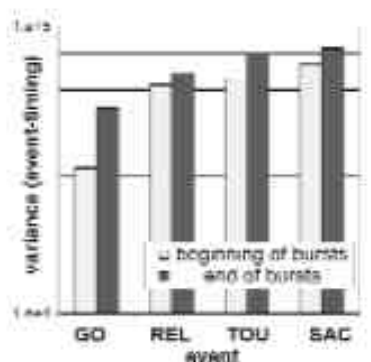

Fig. 5. Search versus repetition differences. Activity of a 'repetition-preferring' cell. (a) The cell discharged primarily in the delay intervals of the ' $d$ ' and ' $e$ ' trials. The activity of the second and fifth intervals (Fig. 1e) was different during the search and repetition periods (ANCOVA, $F 1,23=10.42, p$ $<0.0037 ; F 1,17=4.68, p<0.045$ ). Note the transition of activity between ' $c$ ' and ' $d$ ' trials. (b) Neural activity of intervals 2 and 5 is plotted against the corresponding MT, RT, saccade direction and number of saccades. No significant correlation was found between activity and RT, MT or number of saccades (Spearman test). (c) Variances of neuronal modulation times (vertical bars) during the repetition period relative to the different events. Variances were not significantly different.

(in arcuate cortex), of movement components (in SMA) or the serial order of specific components within a sequence, irrespective of which movements precede or follow (in SMA and pre-SMA) 18-21. In the caudate nucleus, neuronal activity may reflect the serial position of sequence components, irrespective not only of the preceding and following movements but also of the movement that is being performed19. Here we show that the anterior cingulate cortex also encoded the serial order of sequence components, irrespective of which component was performed. Our findings could be compared to those obtained in the ventral striatum, where neuronal activity is specific to parts of the schedule in a predictable series of trials22. Our results may reflect a role for the ACd in encoding sensory or motor events in a predictable sequence of movements according to the timing of their occurrence before the end of the sequence and the reward or goal.

\section{Search versus repetition}

We found that one group of ACd neurons was more active during the search period, whereas another group was more active during the repetition period. For the majority of neurons, these differences could not be explained by differences in the execution of arm movements or saccades between the two periods. These results can be interpreted in two ways. One possibility is that the observed search/repetition

differences may have been related to reward expectation. Activity of repetition-preferring or search-preferring cells would depend on whether the animal did or did not anticipate a reward. There is evidence that movementrelated neurons in the caudate nucleus respond differently for rewarded and non-rewarded movements23. Our data would extend this property to the $\mathrm{AC}$ cortex and to sequences of movements. The other possibility is that the differences relate to a different monitoring of sensory-motor loops in the two periods. Related explanations are given for activity recorded in the pre-SMA during sequential procedural learning and task performance involving updating of motor plans24,25. The alteration of the time relationships between target press and orienting saccades in the two periods supports this interpretation (Fig. 2). Thus searchrelated activities may be specific to a situation requiring flexibility of behavioral responses, evaluation of the outcomes and short-term memory of movements, whereas repetitionrelated activities may be specific to a regime of memory-based motor performance in which errors are unlikely and control less necessary. This interpretation would be consistent with data from human imaging studies and, hence, a functional correspondence between human and monkey ACC.

\section{Anticipation of the solution}

The transition from search-related to repetitionrelated activity was observed as soon as the animal had acquired enough information during the trial-anderror process to predict the correct solution and the reward. This remarkable capacity probably resulted 

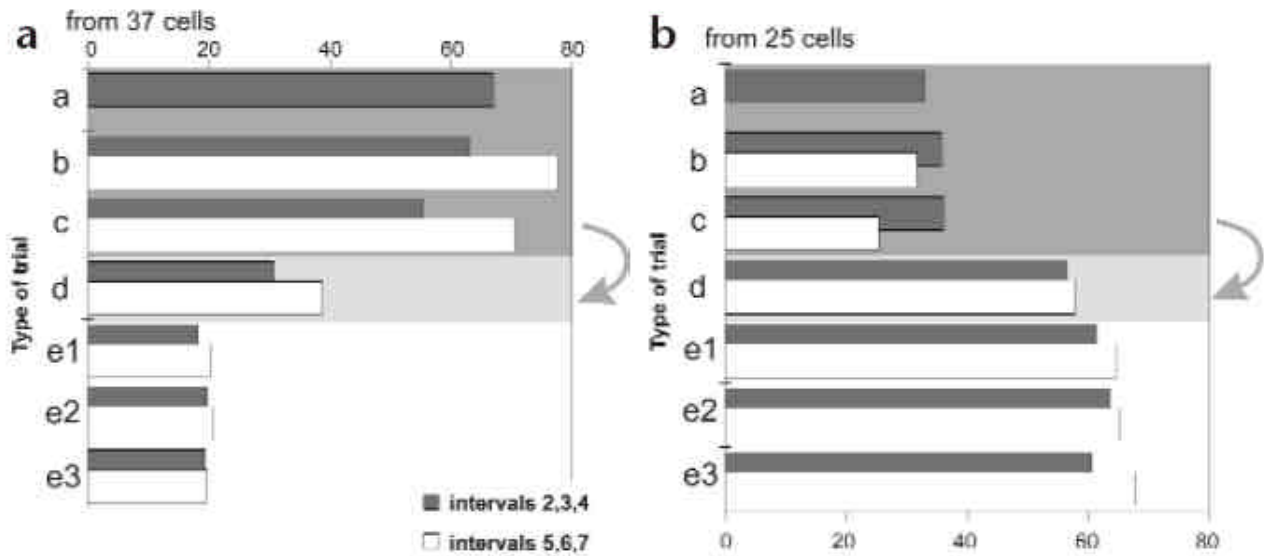

Fig. 6. Population activity and transition from search to repetition. (a) 'Search-preferring' cells; (b) 'repetition-preferring' cells. Pre- and post-acquisition and delay intervals are pooled together. In ordinates, the letters ('a' to 'e') correspond to the different types of trials. Normalized activity is shown on the abscissa. For each cell, the mean activities in each interval are normalized to the maximum ( $\max =100)$ and to the minimum $(\min =0)$ mean across the different types of trials. Then intervals $2,3,4$ and 5, 6, 7 are pooled separately and averaged. The figure illustrates the transition of activity between search and repetition (Mann-Whitney $U$ test. (a) 'c' versus 'd', $p<10-4$; 'd' versus 'e', $p<0.01$. (b) 'c' versus 'd', $p<0.002$; 'd' versus 'e', $p<0.52)$.

from learning, during the training sessions, all the configurations and procedures that directly led from an incorrect sequence in one trial to the solution and the reward in the next. During the search period, as long as the animal had not identified the correct sequence, choices were not made according to a known, unquestionably rewarded goal. Thus, specific cognitive processes must have guided behavior throughout this period until the outcome of a particular choice fulfilled a recognition test, identifying the solution and ending the search period (the 'halting problem' in problem solving 26 ). Neural correlates of this recognition test were not observed, but the transition of neural activity from search to repetition modes may indicate the completion of such a test even before the actual evaluation of the solution.

\section{METHODS}

Animals and materials. Rhesus macaques were trained to sit in a primate chair in front of a tangent touch-screen (Microtouch System, Methuen, USA) coupled to a display monitor located at arm's reach. A computer controlled the presentation on the monitor of visual stimuli, which served as light targets, and recorded the position and correctness of each touch (CORTEX software, NIMH Laboratory of Neuropsychology, Bethesda, Maryland). Eye movements were recorded using the scleral search coil technique. The position of gaze was controlled by a moving eye-position window $\left(12^{\circ}, 12^{\circ}\right)$ centered on the fixation point (FP) or on the different targets. The size of the moving eye fixation window took into account the size of the targets $\left(6^{\circ}{ }^{\circ} 6^{\circ}\right)$ and discrepancies between actual direction of gaze (on the targets) and its measurement by the search-coil technique. A recording cylinder was implanted over the anterior cingulate cortex. Previously described surgical procedures and electrophysiological techniques21 were carried out according to the 1986 European Communities Council Directive (Ministère de l'Agriculture et de la Forêt, Commission Nationale de l'Expérimentation Animale). Behavioral protocol. The task consisted of finding, by trial and error, the correct sequence for touching three fixed spatial targets (Fig. 1). The animal began a trial by touching the lever, which immediately triggered the appearance of the FP on the screen. The animal was required to fixate its gaze on the FP, which then remained illuminated for $2 \mathrm{~s}$; $800 \mathrm{~ms}$ after the onset of fixation, all targets were simultaneously illuminated at the standard level. After the FP was extinguished, the animal was required to make a saccade to the first target within 600 ms. After a period of fixation on the first target (900-1200 ms), all targets turned white

('GO' signal), and the animal had to release the lever and touch the same (fixated) target during the subsequent 1000 ms. If the touch was correct, all three targets reverted to standard illumination while the monkey maintained its hand position and saccaded to the second target. Following another period of fixation, the targets turned white again, and the animal had to touch the second target. Acquisition of the third target followed the same rule. Oculomotor activity was free in the time period between target release (by the hand) and $600 \mathrm{~ms}$ after the target press. A correct trial was defined as three touches performed in the correct order and was rewarded with a drop of juice given at the end of that trial. The sequence was then repeated until the animal had performed a total of four correct trials (the first correct trial and three repetitions; Fig. 1c). When the repetition period was terminated, a central red circle was flashed three times, and a tone was delivered to indicate a new sequence. Following an incorrect touch, a break of fixation or a premature touch, all targets were extinguished, and the trial was aborted. The monkey then had to resume the search from the beginning. There were six possible sequences ( $L-$ $R-U, L-U-R, U-L-R, \quad U-R-L, R-L-U$ or $R-U-L)$. The successive sequences were chosen randomly with the condition that successive sequences never had the same first target. During each $2-3 \mathrm{~h}$ recording session, neural activity was recorded while the animal performed the problem-solving task. Units showing clear changes in firing rate in relation to one or more task events were selected for on-line storage in digital form (resolution, $1 \mathrm{~ms}$ ).

Data analyses. In each single trial, times of lever- or target-release (REL), target-touches (TOU) and saccade onsets were measured and stored. Hand-reaction times (RTs), movement times (MTs), saccade latency (SLAT), number of saccades (NB) and direction of saccades (DIR) between successive targets were computed. (In monkey 2, oculomotor activity could not be controlled during the testing sessions.) Times of neuronal modulation (from the beginning of the first burst to the end of the last burst) were determined for each trial by a Poisson spiketrain analysis25. Significance level was set at $p<0.01$. Relation of the modulation to an 
event (GO, REL, TOU or SAC) was determined by comparing the variances of the neuronal-modulation times relative to these events with an F-test. The F-ratio was calculated by dividing the larger variance by the smaller variance. If this ratio was significant (at $p<0.01$ ), then the neuronal modulation was considered to be related to the event(s) that had the least variance. Rasters and peri-event histograms were constructed for all recorded neurons (using PCOFF Software, NIMH Laboratory of Systems Neuroscience, Bethesda, Maryland). The level of neuronal activity was categorized by the epoch and interval in which it occurred (see Results; Fig. 1e). The three types of interval were considered as separate cases. A cell might show task-related activity in more than one interval. Trial-by-trial firing rates were computed for each interval in the three epochs and were the basis of the statistical analysis. The reference was the $500 \mathrm{~ms}$ period preceding onset of the targets. Interval-specific activity during correct trials was tested with a oneway ANCOVA $(p<0.05)$ for relatedness to ordinal position (three epochs; E1, E2, E3) and to spatial variables associated with the hand movement (target of origin and targeted endpoint of the movement). RTs and MTs were used as covariates to remove a possible effect of these parameters on the serial order and the spatial selectivity of the neurons. Because variations in oculomotor behavior could also explain neural activity modulations, covariates associated with saccades (DIR, NB and SLAT) were included in additional analyses performed in 55 cells. To test differences between search and repetition, different types of trials were considered: ' $a$ ', 'b', 'c', 'd' and 'e1-e3' (see main text). We defined a search period (trials $a, b$ and $c$ ) and a repetition period (d, e1, e2 and e3). Activity of the 9 intervals within 1 period was compared with the activity of the corresponding intervals in the other (Mann- Whitney test, $p<$ 0.05). In each interval, activity found to be selective for search versus repetition was also tested using ANCOVA $(p<0.05)$ and including RTs and MTs as covariates. In 55 cells, DIR, NB and SLAT were also included as covariates. C2 goodness-of-fit test was used to compare the relative frequencies of categorized activity. All statistical analyses were performed with Statisticaâ software (StatSoft, Tulsa, Oklahoma).

\section{ACKNOWLEDGEMENTS}

We thank S. Castner, W. Clower, J. Bullier, M. Jeannerod, K. Knoblauch and L. Romanski for their comments and V. Auger, $C$. Baleydier, V. Gaveau, M.L. Loyalle, P. Monjaud and C. Urquizar for technical assistance. This work was supported by GIS sciences de la cognition, France.

\section{Received 10 January; Accepted 17 March 2000}

1. Shallice, T. From Neuropsychology to Mental Structure (Cambridge Univ.Press, 1988)

2. Wise, S. P., Murray, E. A. \& Gerfen, C. R. The frontal-basal ganglia system in primates. Crit. Rev. Neurobiol. 10, 317-356 (1996).

3. Passingham, R. E. Attention to action. Phil. Trans. R. Soc. Lond. B Biol. Sci. 351, 1473-1479 (1996)

4. Paus, T., Petrides, M., Evans, A. \& Meyer, E. Role of the anterior cingulate cortex in the control of oculomotor, manual and speech responses: a positron emission tomography study. J. Neurophysiol. 70, 453-468 (1993).

5. Posner, M. I. \& DiGirolamo, G. J. in The Attentive Brain (ed. Parasuraman, R.) 401-423 (MIT Press, Cambridge, Massachusetts 1998).

6. Jueptner, M. et al. Anatomy of motor learning. I. Frontal cortex and attention to action. J. Neurophysiol. 77, 1313-1324 (1997)

7. Carter, S. C. et al. Anterior cingulate cortex, error detection, and the onlinemonitoring of performance. Science 280, 747-749 (1998).

8. Picard, N. \& Strick, P. L. Motor areas of the medial wall: a review of theirlocation and functional activation. Cereb. Cortex 6, 342-353 (1996).

9. Devinsky, O., Morrell, M. J. \& Vogt, B. A. Contributions of anterior cingulate cortex to behavior. Brain 118, 279-306 (1995).

10. Zilles, K. et al. Mapping of human and macaque sensorimotor areas by integrating architectonic, transmitter receptor, MRI and PET data. J. Anat.

187, 515-537 (1995).
11. Shima, K. et al. Two movement-related foci in the primate cingulate cortex observed in signal-triggered and self-paced forelimb movements. J. Neurophysiol. 65, 188-202 (1991).

12. Shima, K. \& Tanji, J. Role for cingulate motor area cells in voluntary movement selection based on reward. Science $\mathbf{2 8 2}$, 1335-1338 (1998).

13. Procyk, E. \& Joseph, J. P. Problem solving and logical reasoning in the macaque monkey. Behav. Brain Res. 82, 67-78 (1996).

14. Dum, R. P. \& Strick, P. L. The origin of corticospinal projections from the premotor areas in the frontal lobe. J. Neurosci. 11, 667689 (1991).

15. Mattelli, M., Luppino, G. \& Rizzolatti, G. Architecture of superior and mesial area 6 and the adjacent cingulate cortex in the macaque monkey. J. Comp. Neurol. 311, 445-462 (1991).

16. Gabbot, P. L. \& Bacon, S. J. Local circuit neurons in the medial prefrontal cortex (areas 24a,b,c, 25 and 32) in the monkey: I. Cell morphology and morphometrics. J. Comp. Neurol. 364, 567-608 (1996).

17. Carpenter, A. F., Georgopoulos, A. P. \& Pellizzer, G. Motor cortical encoding of serial order in a context-recall task. Science 283, 1752-1757

(1999).

18. Barone, P. \& Joseph, J. P. Prefrontal cortex and spatial sequencing in macaque monkey. Exp. Brain Res. 78, 447-464 (1989).

19. Kermadi, I. \& Joseph, J. P. Activity in the caudate nucleus of monkey during spatial sequencing. J. Neurophysiol. 74, 911-933 (1995).

20. Tanji, J. \& Shima, K. Role for supplementary motor area cells in planning several movements ahead. Nature 371, 413-416 (1994).

21. Clower, W. T. \& Alexander, G. E. Movement sequence-related activity reflecting numerical order of components in supplementary and presupplementary motor areas. J. Neurophysiol. 80, 15621566 (1998)

22. Shidara, M., Aigner, T. G. \& Richmond, B. J. Neuronal signals in the monkey ventral striatum related to progress through a predictable series of trials. J. Neurosci. 18, 2613-2625 (1998).

23. Schultz, W., Tremblay, L., Hollerman, J. R. \& Mirenowicz, J. in The Association cortex. Structure and Function (eds. Sakata, H., Mikami, A. \& Fuster, J. M.) 67-84 (Harwood, Amsterdam, 1997).

24. Nakamura, K., Sakai, K. \& Hikosaka, O. Neuronal activity in medial frontal cortex during learning of sequential procedures. $J$. Neurophysiol. 80, 2671-2687 (1998).

25. Shima, K., Mushiake, H., Saito, N. \& Tanji, J. Role for cells in the presupplementary motor area in updating motor plans. Proc. Natl. Acad. Sci. USA 93, 8694-8698 (1996).

26. Miller, G. A., Galanter, E. \& Pribram, K. H. Plans and the Structure of Behavior (eds. Miller, G. A., Galanter, E. \& Pribram, K. H.) 159175 (Adams- Bannister-Cox, New York, 1986).

27. Hanes, D. P., Thompson, K. G. \& Schall, J. D. Relationship of presaccadic activity in frontal eye field and supplementary eye field to saccade initiation in macaque: Poisson spike train analysis. Exp. Brain. Res. 103, 85-96 (1995). 